\title{
Pre-University OUTREACH Through a SATEllite Design Competition
}

\author{
Dario Schor ${ }^{l}$, Kane Anderson ${ }^{l}$, Mohammadreza Fazel Darbandi ${ }^{l}$, Greg Linton ${ }^{1,8}$, Matthew Woelk, \\ Cody Friesen ${ }^{2}$, Scott McKay ${ }^{2,3}$, Katriana Soriano ${ }^{4}$, Chad Stasiuk ${ }^{5}$, Johnson Vilayvanh ${ }^{1}$, \\ Brendan Cade ${ }^{5}$, David Terrazas ${ }^{1}$, Troy Denton ${ }^{1}$, Stephanie Reid ${ }^{6}$, \\ Witold Kinsner ${ }^{1}$, M.D.(Ron) Britton ${ }^{7}$, Dave Weichel ${ }^{8}$, Debbie Leiter ${ }^{9}$, Rob Striemer ${ }^{10}$, and Norman Lee ${ }^{11}$ \\ ${ }^{2}$ Dept. of Physics \& Astronomy, ${ }^{3}$ Dept. of Cemistry, Faculty of Science, \\ ${ }^{4}$ University 1, Faculty of Arts, ${ }^{7}$ Dept. of Marketing, Asper School of Business, \\ University of Manitoba, Winnipeg, MB, Canada R3T 5V6 \\ ${ }^{8}$ Canadian Forces School of Aerospace Studies, Winnipeg, MB, Canada \\ ${ }^{9}$ Centre of Excellence Initiative, Canadian Manufacturers \& Exporters, Winnipeg, MB, Canada \\ ${ }^{10}$ Shaftesbury High School, Winnipeg, MB, Canada \\ ${ }^{11}$ MindSet, Government of Manitoba, Winnipeg, MB, Canada \\ \{dschor|kinsner\}@ece.umanitoba.ca
}

\begin{abstract}
The Engineering Profession is seen as a holistic discipline affecting many areas of everyday life. Even though practicing professionals would not dispute the statement, it is often hard to convey the idea to preuniversity students, as it appears overwhelming and presumptuous. Examples comprising of many different subjects such as bridges, airplanes, and computers, are used to reduce the anxiety. But, these examples are part of everyday life and thus fail to inspire a new generation of engineers. To overcome this problem, the University of Manitoba Space Applications and Technology Society is using a student-designed nano-satellite, T-Sat, as a means to promote the profession and motivate a new generation by making space accessible to undergraduate and graduate students. This paper describes the outreach presentations and hands-on workshops organized through a satellite design competition that have reached more than 3,000 pre-university students, university students, and industry professionals between January 2011 and May 2012.
\end{abstract}

Keywords: Pre-university outreach; Lifelong learning.

\section{INTRODUCTION}

The website "Why Engineering" developed by Engineers Canada answers the fundamental question by saying, "engineering is all around us" and then list a series of outcomes from engineering projects such as bridges, airplanes, telephones, and computers [1]. Even though practicing professionals would not dispute the definition, those currently not in the field, and in particular pre-university students, high school teachers, high school counselors, and parents, can find the concept overwhelming. How can a single person do or prepare for all these things? The holistic view is well suited for mature individuals that comprehend how fundamental principles can be applied to multiple disciplines and the collaborative process that makes these examples of engineering projects feasible.

Furthermore, although it is easy to relate to the examples of products selected by Engineers Canada, these do not motivate today's generation in the same way other events and technologies inspired previous generations. Although there are new developments in the design of all the examples selected, these are considered everyday items for todays generation, and thus fail to inspire young minds in the same way that Alcock and Brown's first nonstop transatlantic flight did in 1919 or the way the space race captivated audiences in the 1960's. The state-of-theart examples inspired previous generations to imagine new technologies and pursue them through careers in engineering, science, and technology.

The University of Manitoba Space Applications and Technology Society (UMSATS) is using a studentdesigned nano-satellite (T-Sat) as a gateway into the holistic engineering profession and a means of inspiring a new generation of students. Although satellites are somewhat an old technology that has been around since Sputnik was launched in 1957, the project demystifies the idea that space is reserved for a few special individuals known as "rocket scientists." The daring nature behind students designing satellites, conducting experiments in space, and taking risks is an excellent venue to inspire a new generation to think of what they could do in the future. In addition, complex systems, such as a satellite, are excellent projects to describe the impact of many different disciplines by focusing on computational, 
electrical, mechanical, or other elements thus providing some elements of the holistic profession.

This paper presents a strategy for outreach developed around the T-Sat mission at the University of Manitoba (UofM). Examples of various activities to different audiences are described to show the impact of and reach of a satellite project as a means of inspiring a new generation of engineers.

\section{T-SAT PROJECT OVERVIEW}

The T-Sat project is the University of Manitoba's entry into the Canadian Satellite Design Challenge (CSDC). This national competition was introduced in 2010 by Geocentrix Inc. to design, build, and test an operational nano-satellite [2]. The competition ends in September 2012, after which the winning design will be launched into low-earth-orbit. The CSDC gives students opportunities to build on the skills acquired in university courses through hands-on experience designing and managing a complex project. The UofM team consists of more than 100 undergraduate and graduate registered students from Engineering, Science, Business, Architecture, and Art working together on various aspects of the project [3]. They are supported by more than 50 advisors from academia, industry, business, military, and government that provide valuable feedback throughout the project.

The spacecraft carries two scientific payloads. The primary payload studies the behavior of a colony of tardigrades (waterbears) when exposed to the harsh space environment. The secondary payload is a remote sensing experiment that gathers information from the Sun through spectroscopy.

The satellite bus consists of all the necessary components to control the experiments and communicate the results to the ground. The bus is $10 \times 10 \times 30 \mathrm{~cm}^{3}$ and has a maximum mass of $4 \mathrm{~kg}$ that host the following six units. The Attitude Determination and Control (ADC) unit uses sun sensors and the Earth's magnetic field to orient the satellite while in orbit to point at the Sun for the spectroscopy experiment. The Communications (COM) unit uses amateur radio frequencies and protocols to establish two-way communication between the satellite and the UofM Satellite Ground Station [4]. Telemetry packets, commands and other on-board operations are managed by the Command and Data Handling (CDH) unit. A Power (PWR) unit harvests power from solar panels into a battery. An active Thermal (THM) unit maintains the temperature of components within their operating ranges and the Structure (STR) houses all the components.

The technical components for the project are augmented with a CSDC requirement that all teams must make at least five presentations per calendar year to different audiences. This initial motivation served to ignite the outreach efforts for the UofM team. Additionally, the team must approach industry for monetary donations, in-kind donations, and access to specialized equipment to help fund the satellite design and related activities.

\section{TYPES OF OUTREACH}

For many organizations, outreach activities are a means of recruiting new students into a program. However, recruitment is simply a subset of outreach activities intended to expose audiences both external and internal to the University to projects, research activities, and opportunities. Such ambitious objectives are accomplished through a combination of comprehensive activities tightly integrated to provide both a high level exposure to certain topics and the necessary depth to make the experience memorable for the audience. In the case of the T-Sat project, students from UMSATS identified five categories for outreach with increasing levels of engagement: seminars, presentations, collaborations, demonstrations, and workshops. All these activities are customized for a particular audience with an appropriate set of learning objectives as described below both conceptually and through examples.

\subsection{Seminars}

Seminars are small lectures on topics of interest that relate to the overall objective and are generally delivered by guest expert speakers. In the case of T-Sat, a seminar entitled "Canada in Space" was delivered by Dr. Nicole Buckley, Chief Scientists of the Canadian Space Agency to a large crowd of university students from the faculties of Science and Engineering. The talk is preceded by a short introduction of the speaker linking their visit to the project being conducted at the university.

Seminar speakers are not directly linked to the project or institution, therefore, the level of engagement from these outreach activities is very low. Therefore, the objective of the talks is to expose the audience to a number of projects through a public figure in order to inspire them to think of their own experiments and projects for the future.

\subsection{Presentations}

Presentations delivered by representatives from the TSat project are aimed at providing an overview of the project with appropriate levels of detail for the target audience as described below.

Presentations to Elementary Schools and Middle Years: In presentations to young audiences, the objective is to provide an exposure to things that they may not have seen 
at home or school. In these cases, using a satellite example insights very interesting discussions blur the lines between reality and science fiction enough to inspire students to think of what they would could do in similar projects.

The presentations to young audiences are often hard to prepare because one cannot assume much about the previous knowledge in the subject. Prompting, theatrics, games, familiarity with cultural trends, and a good sense of humor are essential to make an impact in a short period of time.

Prompting allows the presenters to gauge the prior knowledge from the students. Simple questions used in TSat presentations included, (i) what are satellites? (ii) what are satellites used for? (iii) can you name a satellite already in space? These questions were selected as they reveal the what, why, and some extent of their exposure to space science and engineering.

Theatrics and games go hand-in-hand as a means for students to understand some complex concepts. For example, in order to describe attitude determination in space to grade 6 students at Luxton School, the UMSATS representatives asked for two volunteers. The first volunteer playing the satellite was blindfolded and disoriented by spinning a few times. Then, a second volunteer would serve as a target used its voice to direct the satellite actor of where to go. This exercise is very short, but serves to prompt the students of the difficulties in navigating and then relating those ideas to the satellite, thus providing an analogous point of reference for attitude determination.

Some knowledge of cultural trends and a good sense of humor are required to answer questions and keep the presentation interesting. The relationship between space projects and science fiction often appears in discussions and it is important not to dismiss these ideas, as those intriguing and innovative ideas can serve as motivation for future projects.

Presentations to High School: With high school audiences, the presentation of the satellite is a venue for many other discussions about university life. The objectives are to (i) provide an overview of the satellite project, (ii) demonstrate how the subject areas studied in high school relate to the satellite, and (iii) address concerns about the transition to university. Thus, the presentations aim to provide further motivation for students to pursue post-secondary education and expose them to different career options as extractions to the satellite project (i.e., engineering, science, and business).

In order to achieve these objectives, the presenters describe some of the challenges in space and then ask questions such as "what are some options to address this problem?" Many of the answers are followed-up by a number of quick questions on implementation, equipment needed, cost, and other constraints that reveal the feasibility of the proposed solution and the scope of the problem. Ultimately, this approach improves the quality of further responses as students begin to consider a number of other criteria that they did not think of prior to that time.

For example, after presenting the importance of attitude determination and control to a Grade 11 Physics class at Vincent Massey Collegiate, the question of what can you use to control the attitude was asked to the students. Immediately, students responded with thrusters leading to follow-up questions on mass for fuel for a minimum 1 year of operations. Another popular answer is shifting weights, which rather than dismissing is interpreted as using a gravity boom to orient the satellite, however, this is only a partial solution when 3-axis stabilization are required. Finally, the students were presented with the concept of torque rods used in T-Sat and the ideas of fields were related to their exposure to the right-hand rule from studying electricity.

Using the approach of asking questions embeds the relationships between the design of T-Sat and the subjects they study in high school and opens the door to short blurbs about university courses that expand on that knowledge as required for the project. Thus, using T-Sat as a venue for an open discussion on high school electives for different career options, university life, and work prospects. Pamphlets and promotional material from the faculties is always available at the conclusion of the presentation for those students interested in learning more.

The multi-year nature of the T-Sat project also enables high school seniors to learn about the project in their final year of studies and join the ongoing activities as soon as they enter university. As an example, two students from a local high school (including one of the co-authors for this paper) heard about the project through a presentation and joined the project during the summer before their first year of university. Although they did not have the background for some of the design activities, they were assigned a task that they could do independently that would have a direct and visible contribution to the project.

Presentations at the University: Presentations at the University of Manitoba are designed to promote the project internally and recruit new students. The internal promotion is necessary in order to gain access to laboratories or resources for a student group project and to showcase the accomplishments of the students, while the recruitment efforts helps to bring more members to the team that can contribute on different portions of the project. Although most events address both objectives together, there are a small number of events that are aimed primarily at recruitment at the beginning of each school year.

Unlike presentations to younger audiences who are required to attend by their school, university audiences 
choose to attend the event and are used to formal lecturelike presentations. Therefore, the style for the presentation and material prepared is very different in nature from all other audiences. The presentations provide the motivation for the project, preliminary designs, background information, justification for preliminary designs with some additional details, and implementation plans. Emphasis is on the design elements and theoretical foundation for the designs, which is slightly different from the implementation based focus for industry audiences described in the next subsection. In these presentations, the audience is rarely polled for their knowledge and rather more time is allotted for interaction following questions from the attendees.

For example, in the summer of 2011, UMSATS hosted a three-day series on different elements of the project on the payloads (hosted by the Faculty of Science), engineering design (hosted by the Faculty of Engineering), and business and marketing for space (hosted by the Asper School of Business). Each presentation provided an overview of the project followed by a more in-depth description of activities related to each faculty that were delivered by students from that faculty participating in the T-Sat project. In addition to promoting the project, this served to secure laboratory space and lots of advice from professors wanting to help.

From the recruitment perspective, presentations are excellent ways to entice students to learn more, however, the retention from those presentations is not very high. Many students are eager to participate, but lack the time or commitment required for the project. Although originally discouraged by the results, UMSATS team leaders identified a number of self-contained tasks that needed to be addressed. Thus, if students were not entirely convinced about joining, they could still contribute in a smaller capacity and get more active if they choose to do so at a later time.

Presentations to Industry: Industry presentations are aimed at promoting the project to potential sponsors, current sponsors, and related industries. The presentations provide an overview of the project at a high level, block diagrams of all subsystems, and emphasis on project outcomes and benefits for students. Unlike other groups, industry audiences always inquire about budgets and timelines, therefore these components must be included in every presentation, as audiences want to see the practical feasibility of the project.

For example, UMSATS delivered lunch \& learn presentations to Magellan Bristol Aerospace and Manitoba Hydro. Since most attendees were practicing engineers, the emphasis was on the design, feasibility, and implementation. It is important for the presenters to be honest about vulnerabilities in the design, open issues, and to highlight needs for the team, as often, audience members can assist in these activities. Special arrangements were made to ensure these presentations counted towards Professional Development credits for Engineers-in-Training with the Association of Professional Engineers and Geoscientists of Manitoba (APEGM).

Similarly to high school presentations, promotional material on the faculties and programs is always available at the conclusion of the presentation. This is not necessarily for the audience members, but rather for their relatives, who may be completing high school and trying to decide on their career options.

Presentations to Special Interest Groups: There are many amateur and professional associations that host frequent presentations for their members on areas of interest. For example, the Winnipeg Amateur Radio Club, IEEE Winnipeg Section, and the Royal Astronomical Society of Canada, as well as audiences in various national and international conferences on related topics. The presentations to local groups are aimed at promoting the project, while conference presentations are aimed at highlighting some of the designs and lessons learned to wider audiences.

The presentations to local interest groups are similar in nature to those for industry, but with an emphasis on specific elements that are applicable to the audience. For example, presentations to IEEE emphasize electrical hardware and software designs, while presentations to the Winnipeg Amateur Radio Club put more emphasis on the communications system for the satellite.

\subsection{Collaborations}

In addition to UMSATS specific events, there are a number of collaborations that allow for more one-on-one interactions with pre-university students, pre-university teachers, and parents. These activities include science fairs, summer camps, and pre-university design competitions. The university students' role is often in the form of a mentor or judge, thus providing some time to prompt students with questions on their project and share some insight from their experience with the T-Sat project. This helps to establish common challenges faced in the projects and serves the pre-university students to see how the skills they are gaining through classes and extra curricular projects are applicable later in their careers.

Examples, of activities included serving as judges for the Canadian National Marsville Program [5] in which grade 6 students designed and built life-support systems for life in Mars. As judges, the UMSATS students asked questions and shared their knowledge of space citing examples of things that they were designing and building. Similarly, in the Canadian Manufacturers and Exporters Discovery Program [6] students designed and implemented a model hovercraft. The university students helped as mentors and judges for students thus sharing 
knowledge of design, project management, documentation, and working as a team.

\subsection{Demonstrations}

Demonstrations for high school and university students are effective ways of engaging students in the satellite project. These events take place in a laboratory or room and consist of a short presentation and live demonstration. The presentation differs from others in that it must be very modular in order to fit during key times in the experiment where there is lots of dead waiting time.

In the case of the T-Sat project, the demonstrations took place in the UofM Satellite Ground Station [4] where students tracked near-earth experiments launched by local high schools [7] or summer camps [8]. The activity lasted 2-4 hours, therefore, short presentations with lots of pictures were used at times to demonstrate the value of the experiment and keep people engaged as the high altitude balloon reached altitudes of more than 100,000 feet. The demonstration included a description of the project, the experiment, and the equipment.

It is important to always have spare qualified volunteers at these events to answer questions and keep things moving as high altitude balloons are live experiments and cannot be paused to address audience questions.

\subsection{Workshops}

Workshops are intended to provide hands-on experience on portions of the satellite project to high school students, high school teachers, university students, and industry members. The workshops begin with a short presentation of the satellite that contextualizes the problem to be addressed in the workshop. Then, studentinstructors discuss the theoretical background for the problem before guiding the attendees through the implementation and testing.

UMSATS ran a number of workshops on Matlab, Simulink, orbital mechanics using STK and embedded systems. For all the workshops, one or two senior students prepared the material (such as slides, sample code, and handouts) and delivered the content. Additional students assisted with in the activities by walking around the room to ensure all attendees were able to complete the activities.

The activities for the workshops are best described by separating the pre-university workshops from those aimed at post-secondary students and industry.

In pre-university workshops, students were instructed on how to interface a sun-sensor to a microprocessor to be used for attitude determination and control of the satellite in orbit. The Arduino platform was selected to simplify the activity and students were guided on how to connect the sensors and other passive components using a breadboard before programming their board with code to read the analog sensor. The objective of the workshops was to demystify the hardware-software interaction in the context of the satellite. Students programmed the boards, interfaced a phototransistor, and monitored the readings on a host computer. The sensor selected was easy to interface and understand by students. Testing was done by covering the sensor from the ambient light to see the effect of the readings during an orbital eclipse and students used their cell phones to shine brighter lights to experience the effects of brighter intensity lights. This project is not overwhelming to students, and allows for some easy extensions for more advanced students finishing quickly (i.e., program responses to show when the sun is identified with the sensor).

The workshops are at most three hours long and provide an exposure get students thinking of how to apply these tools and techniques to other projects. The difficulty, is that for many high school students without an electronics or programming background it is sometimes difficult to implement these advanced ideas and can lead to disappointing or overwhelming. Therefore, the workshop must be seen as a starting point for exploring a variety of topics rather than a one-time event. For example, following a series of workshops on Arduino processors, a high school contacted the team with some follow-up questions on interfacing a Global Positioning System (GPS) module to read off coordinates [7]. The workshop instructors answered some of the questions by email and then organized a follow-up workshop to focus on the specific need and build on the knowledge and support the students. In the follow-up workshop, the students successfully interfaced a GPS to an Arduino, read the coordinates, and then programmed their board to turn on an LED when walking through a designated target zone of their football field.

The more experienced audiences used the Texas Instruments MSP430 microprocessor that is being used in the T-Sat project as well as Arduinos with a variety of shields and sensors. The objectives in these cases were to provide exposure to a number of different microprocessors and their integration into a larger system. Industry attendees were young professionals looking to expand their repertoire of skills.

The hands-on nature of the workshops provided for lots of interactions, questions-and-answers, and discussions with all audiences. This helped disseminate information on the project, the faculty, and possible career options within the context of the satellite mission.

\section{BENEFITS OF STUDENT-LED OUTREACH}

Student-led outreach activities are an effective means of promoting the university through the enthusiasm of 
students working on a project like T-Sat. Some of the added benefits of these activities include:

1. Practice presentation skills talking to different audiences, preparing materials, and answering questions;

2. Preparation for answering and presenting to the CSDC judges;

3. Promotion of university and department;

4. Relationship to both young and old audiences;

5. Mentorship from experienced students to novices on how to run the activities and workshops; and

6. Informal learning opportunities for students as they receive feedback on both technical content and presentation skills.

\section{CONCLUDING REMARKS}

This paper presented a comprehensive outreach plan to reach many audiences through a satellite project. Since January 2011, the T-Sat project has reached more than 3,100 people through a combination of activities such as those described in this paper. The nature of the activities are designed to not overwhelm audiences but rather see the scope of activities going on at the University of Manitoba. This serves to entice pre-university audiences to learn more about career opportunities within the context of the presentation, demonstration or workshop. Above all, it provides motivation for studying math, physics, and other subjects to understand nature and create new artifacts.

\section{Acknowledgements}

Special thanks to the following students who, in addition to the authors, volunteered their time for many outreach activities Aaron Yanofsky, Ahmad Byagowi, Arash Fazel-Darbandi, Benson Guest, Benyamin Arzhang, Brady Russell, Craig Nemeth, Emily Bashford, Frank Serafin, Hossain Masroor, Kathryn Marcynuk, Kris Goodmanson, Mahmoud Alzaibak, Matthew Kulasza, Matthew Sebastian, Pawel Glowacki, Ravindi Gunasekara, and Ryan Kehrer.

Special thanks to Amber Skrabek, Janet Premak, and Dr. Jonathan Beddoes from the Faculty of Engineering for supporting the activities and providing funding for the workshop materials.

\section{References}

[1] Why Engineering, 2011. Available as of May 2012 from http://www.whyengineering.ca.

[2]Geocentrix Technologies Ltd., H. R. MacMillan Space Centre, and Jaymie M. Matthews, "A proposal to initiate a satellite design challenge for Canadian universities," Vancouver, BC: Geocentrix, January 13, 2009.

[3]Witold Kinsner, M.D.(Ron) Britton, Dario Schor, Arash Fazel Darbandi, Kris Goodmanson, Cody Friesen, Emily Bashford, "Preliminary experience from the Canadian Satellite Design Challenge," in Proc. of the Canadian Engineeering Educaiton Association Conference, CEEA 2011, (St. John's, NL, Canada, June 6-8, 2011), 2011.

[4] Dario Schor, Witold Kinsner, and Alan Thoren, "Satellite ground station emulator: an architecture and implementation proposal," in Proc. of the IEEE Canadian Conference on Electrical and Computer Engineering, CCECE 2009, (St. John's, NL; May 3-6, 2009), 2009, pp. 874-879.

[5] Canadian National Marsville. Available as of May 2012 from http://www.nrc-cnrc.gc.ca/eng/ education/marsville/index.html.

[6] Debbie Leiter, Patty Whitehill, Larry Leiter, Rae-Lynn Rempel, Bill Noakes, Dario Schor, Andres Chapman, Jeffrey Couch, Brad Wallin, Janet Premak, John Chaput, Jenna Biegun, and Alvina Penner, "Product lifecycle experience for pre-university students through a hovercraft competition," in Proc. of Can. Engineering Education Conf., CEEA12 (Winnipeg, MB: June 17-20, 2012), 2012. (Appearing in these proceedings)

[7] Shaftesbury High Altitude Robotics Project. Available as of May 2012 from http://shsballoonproject.pbworks.com.

[8] Witold Kinsner, M. G. (Ron) Britton, Jeff Cieszecki, Wayne A. Ellis, Alan Thoren, Dario Schor, Brian Statham, Greg Linton, Tom Tessier, Rob Striemer, Colleen Flather, Barbara Bowen, and Norman Lee, "A space adventure camp and preuniversity outreach," in Proc. of Can. Engineering Education Conf., CEEC11 (St. John's, NL: June 6-8, 2011), 2011. 